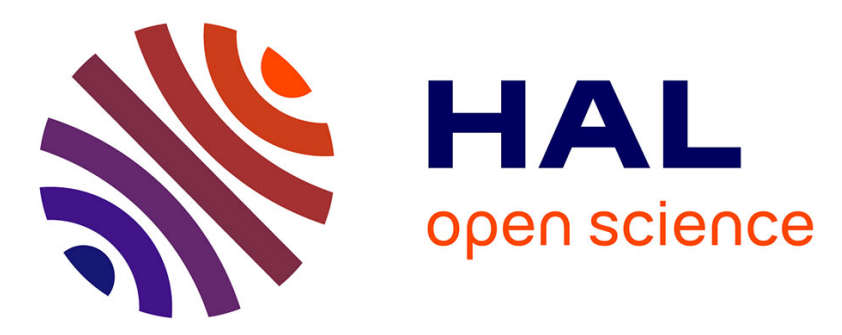

\title{
Three-dimensional trapping and assembly of small particles with synchronized spherical acoustical vortices
}

Zhixiong Gong, Michael Baudoin

\section{To cite this version:}

Zhixiong Gong, Michael Baudoin. Three-dimensional trapping and assembly of small particles with synchronized spherical acoustical vortices. Physical Review Applied, 2020, 14 (6), pp.064002. 10.1103/PhysRevApplied.14.064002 . hal-03329991

\section{HAL Id: hal-03329991 https://hal.science/hal-03329991}

Submitted on 31 Aug 2021

HAL is a multi-disciplinary open access archive for the deposit and dissemination of scientific research documents, whether they are published or not. The documents may come from teaching and research institutions in France or abroad, or from public or private research centers.
L'archive ouverte pluridisciplinaire HAL, est destinée au dépôt et à la diffusion de documents scientifiques de niveau recherche, publiés ou non, émanant des établissements d'enseignement et de recherche français ou étrangers, des laboratoires publics ou privés. 


\title{
Three-Dimensional Trapping and Assembly of Small Particles with Synchronized Spherical Acoustical Vortices
}

\author{
Zhixiong Gong $\odot^{1}$ and Michael Baudoin $\odot^{1,2, *}$ \\ ${ }^{1}$ Univ. Lille, CNRS, Centrale Lille, Yncra ISEN, Univ. Polytechnique Hauts-de-France, UMR 8520-IEMN, \\ F-59000 Lille, France \\ ${ }^{2}$ Institut Universitaire de France, 1 rue Descartes, 75005 Paris, France
}

(Received 16 May 2020; revised 28 September 2020; accepted 23 October 2020; published 1 December 2020)

\begin{abstract}
Three-dimensional harmless contactless manipulation and assembly of micro-objects and microorganisms would open other horizons in microrobotics and microbiology, e.g., for microsystems assembly or tissue engineering. In this paper, we theoretically show that small particles compared to the wavelength can be trapped and assembled in three dimensions with synchronized spherical vortices. The particles can be approached both laterally and axially and, for each configuration, the maximum assembly speed can be determined by balancing the Stokes' drag force and the critical radiation force. These theoretical results provide guidelines to design acoustical tweezers able to trap and assemble particles in three dimensions.
\end{abstract}

DOI: 10.1103/PhysRevApplied.14.064002

\section{INTRODUCTION}

The father of single beam optical tweezers, Arthur Ashkin, was awarded the Nobel prize in physics in 2018 for his contribution to "groundbreaking inventions in the field of laser physics," which opened the door to major breakthroughs in physics including neutral atom trapping and cooling [1], but also the manipulation of objects ranging from atoms [2] to Bose-Einstein condensates [3] or living cells and bacteria [4]. Yet, optical tweezers have stringent limitations in life science, which are prohibitive for many applications. (i) The forces that can be applied with optical tweezers on micro-organisms are limited to typically less than $50 \mathrm{pN} \mathrm{[5]} \mathrm{and} \mathrm{require} \mathrm{high} \mathrm{intensity}$ beams. This is due to the fact that the optical radiation force used to trap objects is proportional to the intensity of the incident field divided by the high value, light speed. (ii) These high intensity beams induce phototoxicity on biosamples due to thermal effects and/or chemical reactions [5-9]. (iii) Finally, objects can only be manipulated in optically transparent media, which prevents their use for most in-vivo applications. The remote contactless manipulation of micro-objects can also be achieved with magnetic tweezers [10,11]. Magnetic tweezers are biocompatible and easy to implement but (i) they can only be used to move magnetic objects [12] or otherwise require pretagging and (ii) they exhibit low trap stiffness and low selectivity.

*michael.baudoin@univ-lille.fr; http://films-lab.univ-lille1.fr/ michael
Many limitations of optical and magnetic tweezers for microbiology applications can be overcome with acoustical tweezers [13]. (i) Since the trapping force applied with acoustical tweezers is, as their optical counterpart, proportional to the incident field intensity divided by the wave speed, the drastically lower speed of sound compared to light leads to trap strengths some orders of magnitude larger than with optical tweezers at the same driving intensity $[14,15]$. (ii) Acoustical tweezers can trap a large variety of materials since only a contrast in density and/or compressibility between the particle and the surrounding medium is required for the acoustic radiation force to exist. (iii) Since acoustic transducers are available from kilohertz to gigahertz frequencies in liquids, the manipulation of particles with sizes ranging from centimeter to nanometer sizes can be envisioned. (iv) Acoustical waves are highly biocompatible for both in-vivo [16] and in-vitro [17-21] applications.

The concept of acoustical tweezers was introduced by Wu et al. in 1991 [22], in analogy with optics. But in this early work, axial trapping was achieved with two counterpropagating beams, hence requiring to position transducers on both sides of the trapping area. Three-dimensional trapping [14], levitation [23], and selective manipulation of microparticles [24] and cells [15] with single beam acoustical tweezers (i.e., with a wavefield synthesis system located on only one side of the manipulation area) has been achieved only recently by using specific wavefields called focused (spherical) acoustical vortices. Indeed, classical focused waves cannot be used as in optics since many particles of interest such as cells or solid particles would be expelled from the focal point. One-sided 
focused acoustical vortices on the other hand provide a three-dimensional (3D) trap for such particles as demonstrated by Baresch et al. [25]. They also enable strong spatial localization of the wavefield, which is required to be selective, i.e., to be able to pick up and move a single particle independently of its neighbors. Beyond the manipulation of individual particles, one key operation in microfluidics and microbiology is to assemble objects. This feature is essential, e.g., to assemble spheroids or for tissue engineering.

A first strategy to move and assemble multiple objects is to dynamically adapt the acoustic field to the target task, as demonstrated in two dimensions by Courtney et al. [26]. Such an adaptive field synthesis strategy nevertheless requires a complex array of transducers that (i) are only presently available at low frequency for relatively large particle trapping and (ii) are opaque, cumbersome, and hence not compatible with classical microscopy environments. In addition, while advanced 3D manipulation of multiple objects has been demonstrated with two arrays of transducers located on both sides of the manipulation area [27], the possibility to trap, move, and assemble in three dimensions multiple objects with single beam acoustical tweezers has not been demonstrated yet. A second strategy is to trap each particle to be assembled at the core of an acoustical vortex and use the interference between synchronized acoustical vortices to assemble them. This possibility has been investigated for the $2 \mathrm{D}$ assembly of small particles compared to the wavelength with cylindrical acoustical vortices by Gong and Baudoin [28]. The advantage of this solution is that it can be implemented for both reconfigurable arrays of transducers [23,26,29$34]$ and static holographic vortex wave synthesis systems $[15,24,29,35-46]$. In this paper, we extend our previous work to the three-dimensional case: we theoretically demonstrate that a particle can be trapped, moved, and assembled in three dimensions using spherical (focused) acoustical vortices of first topological order. While further numerical and experimental investigation is necessary to investigate the case of vortices synthesized with a finite aperture, this work prefigures the use of focused acoustical vortices for assembly of particles with single beam acoustical tweezers.

\section{3D TRAPPING IN A SINGLE SPHERICAL BESSEL ACOUSTICAL VORTEX}

\section{A. Spherical Bessel acoustical vortices}

Cylindrical and spherical Bessel vortices (SBVs) are separate variable solutions of the d'Alembert wave equation in cylindrical and spherical coordinates, respectively [13]. These waves are spinning around a phase singularity wherein the amplitude cancels, surrounded by a ring of high intensity. Cylindrical vortices, investigated in acoustics by Hefner and Marston [29] are laterally focused waves. These waves are interesting to trap particles laterally (see, e.g., Ref. [42]), but they can only push or pull (and not trap) particles along their propagation axis depending on the particles and beam properties [47]. Spherical acoustical vortices on the other hand focus the energy in three dimensions and hence can create a 3D trap even with a finite aperture [25]. Spherical Bessel beams are defined by the equation [13]

$$
p=A_{0} j_{n}(k r) P_{n}^{m}(\cos \theta) e^{i(m \varphi-\omega t)},
$$

where $A_{0}$ is the beam amplitude, $j_{n}(k r)$ is the spherical Bessel function of the first kind of order $n, k=\omega / c_{0}$ is the wavenumber, $\omega$ is the angular frequency, $c_{0}$ is the wave speed, $r$ is the radius, the $P_{n}^{m}(\cos \theta)$ are the associated Legendre polynomials of order $(n, m), \theta$ and $\varphi$ are the polar and azimuthal angles in spherical coordinates, $z$ is the central axis of the vortex, $(x, y)$ are the lateral directions, and $t$ is the time. Finally, $m$ is the topological charge of the spherical Bessel beams verifying $-n \leq m \leq n$, which describes the periodic number of phase change from 0 to $2 \pi$ in the transverse plane. The Bessel functions and associated Legendre polynomials are given in Figs. 1(a) and 1(b), respectively. Spherical Bessel acoustical vortices correspond to Bessel beams of order $m \geq 1$, since in this case the phase is spinning around the $z$ axis. The case $m=0$, however, corresponds to spherically focused waves. As this paper is centered on the long wavelength regime (LWR) $(k a \ll 1$, with $a$ the radius of the trapped object) and in this regime, particles with positive contrast factors (like a solid particle surrounded by a liquid) are expelled (not trapped) from the center of spherically focused waves, we will not further investigate this case. Finally, we note that the average power $\langle\mathcal{P}\rangle$ required to synthesize an acoustical vortex of amplitude $A_{0}$ is proportional to (see Appendix A)

$$
\langle\mathcal{P}\rangle \propto \frac{\pi A_{0}^{2}}{\rho_{0} k \omega} \frac{2(n+m) !}{(2 n+1)(n-m) !} .
$$
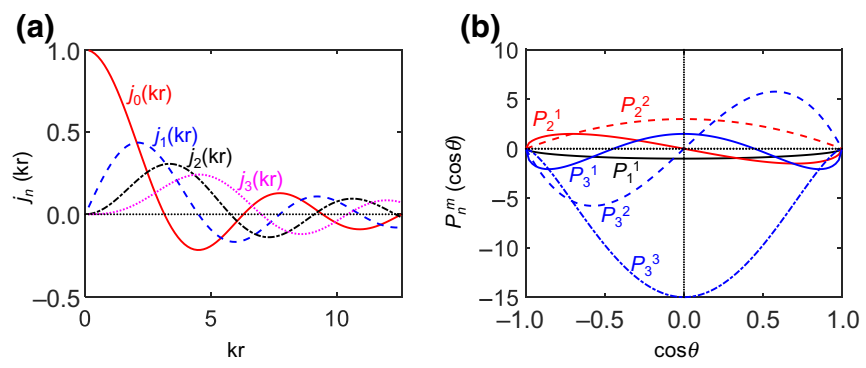

FIG. 1. (a) Spherical Bessel function $j_{n}(k r)$ magnitudes as a function of the dimensionless radial distance $k r$ with $k=\omega / c_{0}$ the wavenumber. (b) Associated Legendre function $P_{n}^{m}(\cos \theta)$ magnitudes as a function of $\cos \theta \in[-1,1]$. 
Hence, to compare the trapping capabilities of acoustic beams of different orders $(n, m)$ at the same input power, we normalize the amplitude $A_{0}$ by $\sqrt{(2 n+1)(n-m) !} / \sqrt{2(n+m) !}$ in the next section.

\section{B. Gradient and scattering forces in the long wavelength approximation}

The force applied on particles much smaller than the wavelength $(k a \ll 1)$ can be calculated with the general formula [14]

$$
\begin{array}{r}
\mathbf{F}=-V_{0}\left\{\nabla\left[f_{1}\left(\frac{|p|^{2}}{4 \rho_{0} c_{0}^{2}}\right)-f_{2}\left(\frac{\rho_{0}|\mathbf{v}|^{2}}{4}\right)\right]\right. \\
-\frac{(k a)^{3}}{3}\left[\left(f_{1}^{2}+\frac{f_{1} f_{2}}{3}\right) \operatorname{Re}\left(\frac{k}{2 c_{0}} p \mathbf{v}^{*}\right)\right. \\
\left.\left.+\frac{f_{2}^{2}}{3} \operatorname{Im}\left(\frac{\rho_{0}}{2} \mathbf{v} \cdot \nabla \mathbf{v}^{*}\right)\right]\right\}
\end{array}
$$

with $V_{0}=4 / 3 \pi a^{3}$ the volume of the particle, $a$ its radius, $f_{1}=\left(1-\kappa_{p} / \kappa_{0}\right)$ the monopolar acoustic contrast factor related to the particle isotropic expansion or compression, $\kappa_{p}$ and $\kappa_{0}$ the compressibility of the particle and fluid, respectively, $f_{2}=3\left(\rho_{p}-\rho_{0}\right) /\left(2 \rho_{p}+\rho_{0}\right)$ the dipolar acoustic contrast factor related to the particle back and forth translation, $\rho_{p}$ and $\rho_{0}$ the density of the particle and fluid, respectively, $p$ and $\mathbf{v}$ the complex pressure and velocity of the incident acoustic fields, and, finally, Re and Im, respectively, denote the real and imaginary parts of a complex number and an asterisk denotes the complex conjugate. This expression can be divided into two main types of contributions: the gradient force $\mathbf{F}_{\text {grad }}$ and the scattering force $\mathbf{F}_{\text {scat }}$.

The force $\mathbf{F}_{\text {grad }}$ constituted by the terms on the righthand side of the first line of Eq. (3) can be expressed as the negative gradient of a potential $U$, known as Gor'kov's potential [48]:

$$
\begin{gathered}
\mathbf{F}_{\text {grad }}=-\nabla U \\
\text { with } U=V_{0}\left[f_{1}\left(\frac{|p|^{2}}{4 \rho_{0} c_{0}^{2}}\right)-f_{2}\left(\frac{\rho_{0}|\mathbf{v}|^{2}}{4}\right)\right] .
\end{gathered}
$$

This gradient force, as its name suggests, results from gradients of the acoustic field magnitude. The first term is a monopolar contribution related to the potential energy density of the acoustic field $\mathcal{V}=|p|^{2} / 4 \rho_{0} c_{0}^{2}$, while the second term is a dipolar contribution related to the kinetic energy density of the acoustic field $\mathcal{K}=\rho_{0}|\mathbf{v}|^{2} / 4$ [49]. We can see from this expression that, when the monopolar acoustic contrast factor $f_{1}$ is positive (i.e., for particles less compressible than the surrounding fluid), particles are pushed by the potential energy toward the nodes (minima) of the pressure field. While, when the dipolar acoustic contrast factor $f_{2}$ is positive (i.e., for particles more dense than the surrounding fluid), particles are pushed toward the antinodes (maxima) of the velocity field. Of course, for plane standing waves, pressure nodes correspond to velocity antinodes, so that particles more dense and less compressible than the surrounding fluid are pushed by both the potential and kinetic energies toward the same location. We will see in the next section that depending on their order $(n, m)$, things are not so obvious for acoustical vortices. Indeed, (i) a SBV is not a standing wave since it is progressive over $\mathbf{e}_{\varphi}$. (ii) Since a SBV is the separate variable solution of the Helmholtz equation in spherical coordinates, its spatial structure is given by the product of three functions depending on $r, \theta$, and $\varphi$, respectively. (iii) The lateral variation is given by a Bessel function (instead of a sin function for plane waves) and the minima of a Bessel function do not coincide with the maxima of its derivative. Hence, since the velocity field is proportional to the gradient of the pressure field, the pressure minima and velocity maxima will not necessarily coincide.

The terms expressed in the second and third lines of Eq. (3) are known as the scattering force $\mathbf{F}_{\text {scat }}$. It contains three terms: one due to the monopolar oscillation of the particle only (proportional to $f_{1}^{2}$ ), one due to the dipolar oscillation only (proportional to $f_{2}^{2}$ ), and one due to crosscoupling between the monopolar and dipolar oscillations (proportional to $f_{1} f_{2}$ ).

It is interesting to note that, for an incident standing wave, only the gradient force acts on the particle, while the scattering force cancels. On the contrary, for a progressive wave, only the scattering force acts on the particle and the gradient force cancels. This can be seen directly from Eq. (3). Note that this analysis is only valid in the LWR $k a \ll 1$. Another interesting point is that the scattering forces are proportional to a factor $(k a)^{3}$, which is very small in the LWR. Hence, generally speaking, scattering forces are weak compared to gradient forces in this regime. As can be seen from Eq. (1), the SBV is neither a standing nor a progressive wave. It is a standing wave over $r$ and $\theta$, while it is progressive over $\varphi$. Hence, we expect a gradient force over $\mathbf{e}_{r}$ and $\mathbf{e}_{\theta}$ and a scattering force over $\mathbf{e}_{\varphi}$, with $\left(\mathbf{e}_{r}, \mathbf{e}_{\theta}, \mathbf{e}_{\varphi}\right)$ the spherical basis. To verify this hypothesis, we can compute the gradient and scattering forces. For the gradient force, we can start by calculating the square of the modulus of the complex pressure and velocity. We obtain

$$
|p|^{2}=p p^{*}=A_{0}^{2} j_{n}^{2}(k r) P_{n}^{m 2}(\cos \theta)
$$

and, since 


$$
\begin{aligned}
\mathbf{v} & =-\frac{i}{\rho_{0} \omega} \nabla p \\
& =-\frac{i A_{0}}{\rho_{0} \omega} e^{i(m \varphi-\omega t)}\left[k j_{n}^{\prime}(k r) P_{n}^{m}(\cos \theta) \mathbf{e}_{r}+\frac{j_{n}(k r)}{r} \frac{d P_{n}^{m}(\cos \theta)}{d \theta} \mathbf{e}_{\theta}+i m \frac{j_{n}(k r)}{r} \frac{P_{n}^{m}(\cos \theta)}{\sin \theta} \mathbf{e}_{\varphi}\right]
\end{aligned}
$$

with $i$ the imaginary unit, we obtain

$$
|\mathbf{v}|^{2}=\frac{A_{0}^{2}}{\rho_{0}^{2} \omega^{2}}\left[k^{2}\left[j_{n}^{\prime}(k r)\right]^{2} P_{n}^{m 2}(\cos \theta)+\frac{j_{n}^{2}(k r)}{r^{2}}\left(\frac{d P_{n}^{m}(\cos \theta)}{d \theta}\right)^{2}+m^{2} \frac{j_{n}^{2}(k r)}{r^{2}} \frac{P_{n}^{m 2}(\cos \theta)}{\sin ^{2} \theta}\right] .
$$

The variations of $|p|^{2}$ and $|\mathbf{v}|^{2}$ in the $(x, y)$ and $(y, z)$ planes are given in Figs. 2(a) and 2(b), respectively, for different orders $(n, m)$. See the Supplemental Material [50] (movie 1) for 3D views of the pressure amplitudes square of the SBVs with different orders for better representation of the entire beam structure. Since the gradient force $\mathbf{F}_{\text {grad }}$ is proportional to the gradients of $|p|^{2}$ and $|\mathbf{v}|^{2}$, which depend only on $r$ and $\theta$ according to Eqs. (6) and (8), we see, as expected, that this force has only nonvanishing components along $\mathbf{e}_{r}$ and $\mathbf{e}_{\theta}$, along which the wave is a standing wave.

Now, we can look at the scattering force. A straightforward calculation gives

$$
\operatorname{Re}\left(p \mathbf{v}^{*}\right)=\frac{m A_{0}^{2}}{\rho_{0} \omega}\left[\frac{j_{n}^{2}(k r)}{r} \frac{P_{n}^{m^{2}}(\cos \theta)}{\sin \theta}\right] \mathbf{e}_{\varphi}
$$

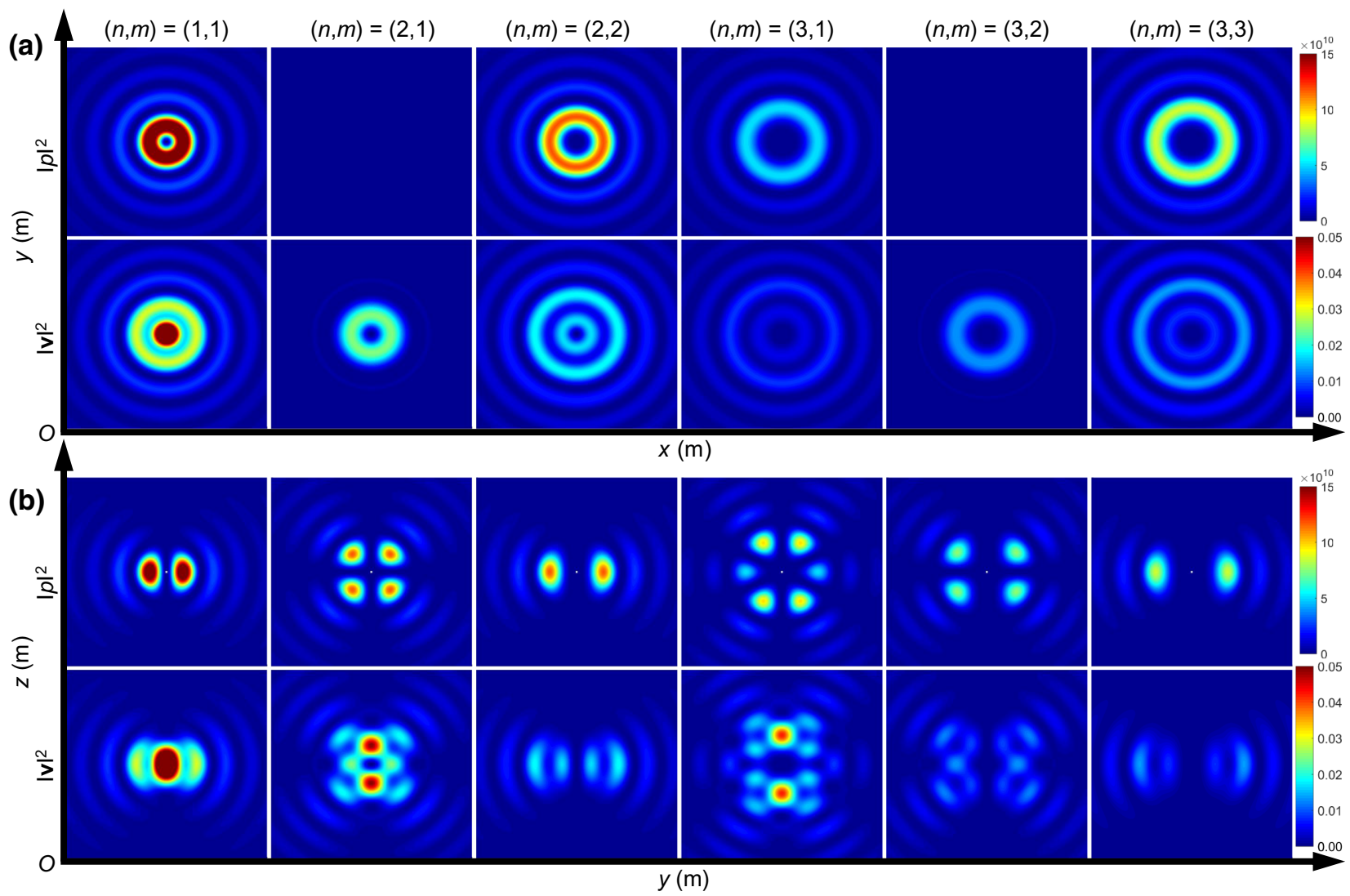

FIG. 2. Square of the pressure modulus $|p|^{2}$ and square of the velocity modulus $|\mathbf{v}|^{2}$ in (a) the $(x, y)$ plane and (b) the (y,z) plane for a single SBV with different orders $(n, m)$ and the same input power [corresponding to an amplitude $A_{0}=10^{6}$ for $(n, m)=(1,1)$ and normalized according to Eq. (2) for other orders]. 
(a)

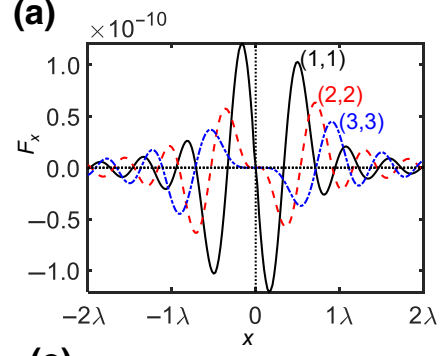

(c)

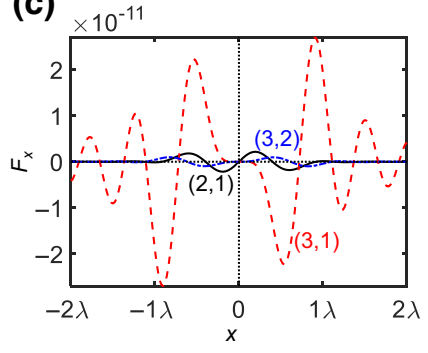

(b)

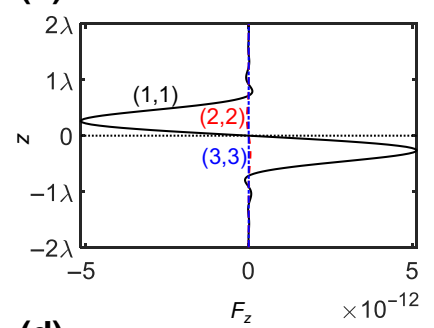

(d)

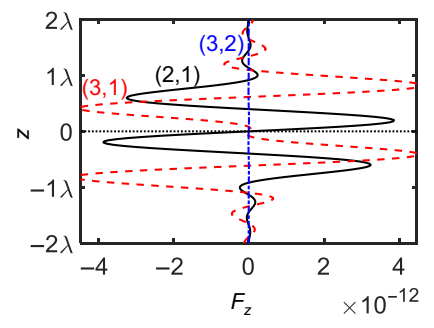

FIG. 3. Gradient lateral force $F_{x}$ and axial force $F_{z}$ exerted on an elastic polystyrene (PS) particle of radius $a=5 \mu \mathrm{m}$ immersed in water by a spherical Bessel vortex of order $(n, m)$ at driving frequency $5 \mathrm{MHz}$. (a),(c) Lateral forces for the orders $(n=m)$ and $(n \neq m)$, respectively. (b),(d) Axial forces for the orders $(n=$ $m)$ and $(n \neq m)$, respectively.

and

$$
\begin{array}{r}
\operatorname{Im}\left(\mathbf{v} \cdot \nabla \mathbf{v}^{*}\right)=\left[v_{r} \frac{\partial v_{\varphi}^{*}}{\partial r}+\frac{1}{r}\left(v_{\theta} \frac{\partial v_{\varphi}^{*}}{\partial \theta}+\frac{v_{\varphi}}{\sin \theta} \frac{\partial v_{\varphi}^{*}}{\partial \varphi}\right.\right. \\
\left.\left.+v_{r} v_{\varphi}^{*}+\frac{v_{r} v_{\varphi}^{*}}{\tan \theta}\right)\right] \mathbf{e}_{\varphi}
\end{array}
$$

where $\left(v_{r}, v_{\theta}, v_{\varphi}\right)$ are the components of the complex acoustic velocity over $\left(\mathbf{e}_{r}, \mathbf{e}_{\theta}, \mathbf{e}_{\varphi}\right)$, respectively, given by Eq. (7). The scattering force is, as expected, directed toward the only direction wherein the wave is progressive, i.e., $\mathbf{e}_{\varphi}$. Since this scattering force is small compared to the gradient force as discussed previously, we neglect it in the subsequent analysis.

\section{Lateral force $[(x, y)$ plane $]$}

In this subsection we analyse the ability of a SBV to laterally trap a particle with positive contrast factors $f_{1}$ and $f_{2}$ at the center of the vortex [i.e., in the $(x, y)$ plane at the altitude $z=0$ ] depending on the order of the vortex $(n, m)$ (with $m>0)$. The $(x, y)$ plane corresponds to $\theta=$

TABLE I. Acoustic properties.

\begin{tabular}{lcccc}
\hline \hline Material & $\begin{array}{c}\text { Density } \rho_{0} \\
\left(\mathrm{~kg} / \mathrm{m}^{3}\right)\end{array}$ & $\begin{array}{c}\text { Compressibility } \\
\kappa(1 / \mathrm{TPa})\end{array}$ & $\begin{array}{c}\text { Longitudinal } \\
\text { speed of } \\
\text { sound } c(\mathrm{~m} / \mathrm{s})\end{array}$ & $\begin{array}{c}\text { Viscosity } \\
(\mathrm{mPa})\end{array}$ \\
\hline PS & 1050 & 172 & 2350 & $\ldots$ \\
Water & 998.2 & 456 & 1482 & 1.002 \\
\hline \hline
\end{tabular}

$\pi / 2$, i.e., $\cos (\theta)=0$. Since $P_{n}^{m}(-x)=(-1)^{(n+m)} P_{n}^{m}(x)$, if $(n+m)$ is odd then $P_{n}^{m}$ is an odd function and $P_{n}^{m}(0)=0$.

Case $A:(n+m)$ is odd. In this case, since $|p|^{2} \propto$ $P_{n}^{m 2}(\cos \theta)$ [see Eq. (6)], the potential energy is null. This can be seen in Fig. 2(a) for $(n, m)=(2,1)$ and $(3,2)$. Hence, the potential energy does not contribute to lateral forces. On the other hand, only the term $\left[d P_{n}^{m}(\cos \theta) / d \theta\right]^{2}$ does not vanish in the expression of the kinetic energy [Eq. (8)]. If we introduce the classical relation

$$
\begin{aligned}
& \frac{d}{d \theta} P_{n}^{m}(\cos \theta) \\
& \quad=\frac{1}{\sin (\theta)}\left[n \cos \theta P_{n}^{m}(\cos \theta)-(n+m) P_{n-1}^{m}(\cos \theta)\right],
\end{aligned}
$$

we see that, when $(n+m)$ is odd, only the term $P_{n-1}^{m}[\cos (\theta)]$ does not vanish when $\theta=\pi / 2$. Thus, the potential becomes

$$
U=-\frac{V_{0} f_{2} A_{0}^{2}}{4 \rho_{0} \omega^{2}} \frac{j_{n}^{2}(k r)}{r^{2}}(n+m)^{2} \frac{P_{n-1}^{m}{ }^{2}(\cos \theta)}{\sin ^{2} \theta} .
$$

In this expression the radial variation is given by the function $j_{n}^{2}(k r) / r^{2}$ with $n \geq 2$, which becomes minimum (null) when $r \rightarrow 0$ [see Fig. 1(a)]. Hence, since $U$ is negative for particles with positive acoustic contrast factor $f_{2}$ (i.e., particle more dense than the surrounding fluid), and $\mathbf{F}_{\text {grad }}=$ $-\nabla U$, such particles are expelled from the center of the vortex when $(n+m)$ is odd. This can indeed be seen in Fig. 3(c) representing the lateral force $F_{x}$ for an elastic polystyrene particle of radius $a=5 \mu \mathrm{m}$ immersed in water and insonified with a SBV at the driving frequency $5 \mathrm{MHz}$. The properties used for the simulation are summarized in Table I and are used for all the simulations presented in this paper.

Case B: $(n+m)$ is even. When $(n+m)$ is even, we can distinguish two cases. For $(n, m)=(1,1)$, both the potential energy and kinetic energy contribute to trap particles with positive contrast factors $f_{1}$ and $f_{2}$ at the vortex center. Indeed, the potential energy is minimum at the center, while the kinetic energy is maximum (see Fig. 2). For the cases $(n, m) \neq(1,1)$, we can see from Fig. 2 that both the potential and kinetic energies are minimum at the center of the vortex. Hence, the potential energy contributes to the lateral trap, while the kinetic energy tends to expel particles with positive contrast factor $f_{2}$ from the center. Hence,

TABLE II. Lateral force for positive contrast factors.

\begin{tabular}{lcc}
\hline \hline & $\begin{array}{c}\text { Potential } \\
\text { energy }\end{array}$ & $\begin{array}{c}\text { Kinetic } \\
\text { energy }\end{array}$ \\
\hline$(n+m)$ odd & No force & Expel \\
$(n+m)$ even, $(n, m)=(1,1)$ & Trap & Trap \\
$(n+m)$ even, $(n, m) \neq(1,1)$ & Trap & Expel \\
\hline \hline
\end{tabular}


depending on whether the compressibility or density contrast is larger, and then the respective magnitudes of the kinetic and potential energy gradients, the particle can either be trapped or expelled.

\section{Axial force (z direction)}

In this subsection we analyse the ability of a SBV to axially trap (along the $z$ axis) a particle with positive contrast factors $f_{1}$ and $f_{2}$ at the center of the vortex, depending on the order of the vortex $(n, m)$ (with $m>0$ ). The $z$ axis corresponds to $\theta=\{0, \pi\}$, i.e., $\cos (\theta)= \pm 1$. Because of the phase singularity on this axis, $P_{n}^{m}( \pm 1)=0$ for all $m \neq 0$ [see Fig. 1(b)]. Hence, from Eqs. (6) and (8), we see that, for $m \geq 1$,

$$
|p|^{2}=0, \quad|\mathbf{v}|^{2}=\frac{A_{0}^{2}}{\rho_{0}^{2} \omega^{2}} \frac{j_{n}^{2}(k r)}{r^{2}}\left(\frac{d P_{n}^{m}(\cos \theta)}{d \theta}\right)^{2}
$$

on the $z$ axis. We can now use the following formula to compute the derivative of the associated Legendre polynomial:

$$
\frac{d}{d \theta} P_{n}^{m}(\cos \theta)= \begin{cases}-\frac{1}{2}\left[(n+m)(n-m+1) P_{n}^{m-1}(\cos \theta)-P_{n}^{m+1}(\cos \theta)\right] & \text { for } 1 \leq m \leq n-1 \\ -\frac{1}{2}\left[(n+m)(n-m+1) P_{n}^{m-1}(\cos \theta)\right] & \text { for } m=n\end{cases}
$$

In this formula, $P_{n}^{m+1}$ is always equal to 0 on the $z$ axis [since $(m+1)>0$ ], while $P_{n}^{m-1}$ is not equal to 0 , only for $m=1$. Thus, only SBV of topological order $m=1$ can exert an axial force. This can be seen in Figs. 3(b) and 3(d). In addition, the radial evolution of the vortex is given by the function $j_{n}^{2}(k r)$, which is maximum at the center $r=$ 0 only for $n=1$ and minimum (zero) otherwise $(n \geq 2)$. This means that only the SBV of order $(n, m)=(1,1)$ can trap a particle axially in the LWR.

\section{E. Summary of Sec. II}

The results of this section are summarized in Tables II and III. This analysis shows that, for particles with positive contrast factors $f_{1}$ and $f_{2}$, a stable lateral trap can only be produced by a SBV of order $(n, m)$ when $(n+m)$ is even. For $(n, m)=(1,1)$, both the potential and kinetic energies contribute to the trap, while for $(n+m)$ even and $(n, m) \neq(1,1)$, the potential energy contributes to the trap while the kinetic energy tends to expel the particle. In addition, $(n, m)=(1,1)$ produces the strongest lateral gradients and hence lateral trapping force, as can be seen in Fig. 3(a). Concerning the axial trap, only the kinetic energy contributes to the axial force and a trap is only obtained for $(n, m)=(1,1)$, which is the only SBV wherein the kinetic energy is maximum at the center. Hence, since only the SBV of order $(n, m)=(1,1)$ enables us to obtain both a

TABLE III. Axial force for positive contrast factors.

\begin{tabular}{lcc}
\hline \hline & Potential energy & Kinetic energy \\
\hline$m \neq 1$ & No force & No force \\
$(n, m)=(1,1)$ & No force & Trap \\
$m=1, n>1$ & No force & Expel \\
\hline \hline
\end{tabular}

lateral and axial trap, we mainly consider this case in the remaining part of this work. Note that the results obtained here for positive contrast factors presented in Tables II and III can be extended to negative contrast factors by simply inverting the "trap" and "expel" words in Tables II and III.

\section{3D PARTICLE ASSEMBLY WITH SYNCHRONISED SPHERICAL BESSEL VORTICES}

\section{A. Synchronized spherical Bessel vortices}

The ability to trap a particle both laterally and axially in the LWR with a SBV of order $(1,1)$ has been demonstrated in the previous section. Note that three-dimensional trapping of a PS particle with a one-sided spherical vortex of order $(1,1)$ beyond the long wavelength approximation has been demonstrated both theoretically and experimentally in Refs. [14,25], while lateral trapping has been demonstrated at micrometric scales in Refs. [15,24]. However, a central question is how to assemble in three dimensions particles with spherical vortices since the ring surrounding the trapped particle is repulsive for a particle located outside (as can be seen in Fig. 3). In our previous work, we theoretically demonstrated the ability to assemble in two dimensions particles trapped at the core of two cylindrical vortices by using their interference, which creates an attractive path between the two vortices. We now examine the $3 \mathrm{D}$ case with spherical vortices.

Consider two SBVs separated by a lateral offset $2 s$ and an axial offset $z_{0}$, as shown in Fig. 4(a). The acoustic pressure perturbation of each individual beam can be described by the formula

$$
p_{j}=A_{j} j_{n}\left(k r_{j}\right) P_{n}^{m}\left(\cos \theta_{j}\right) e^{i m \varphi_{j}} e^{i \beta_{j}},
$$




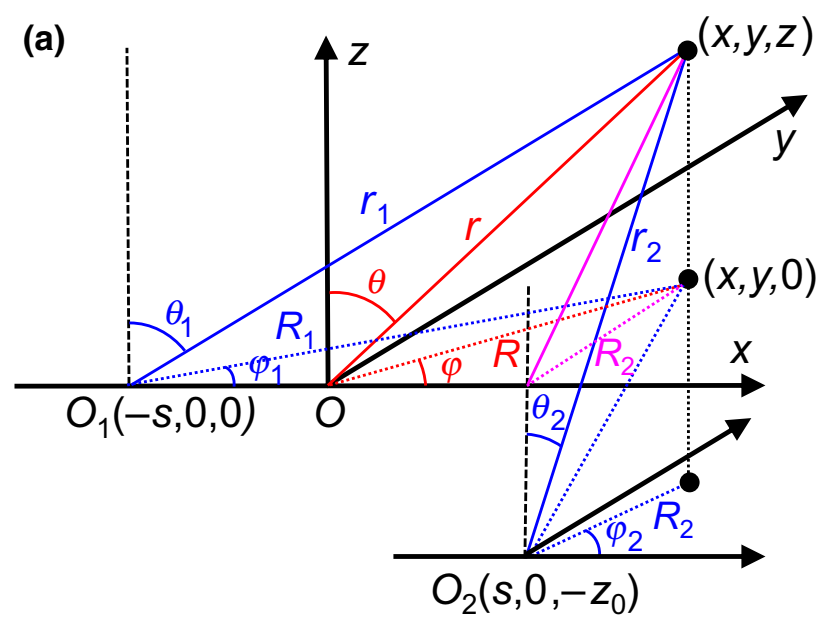

(b)

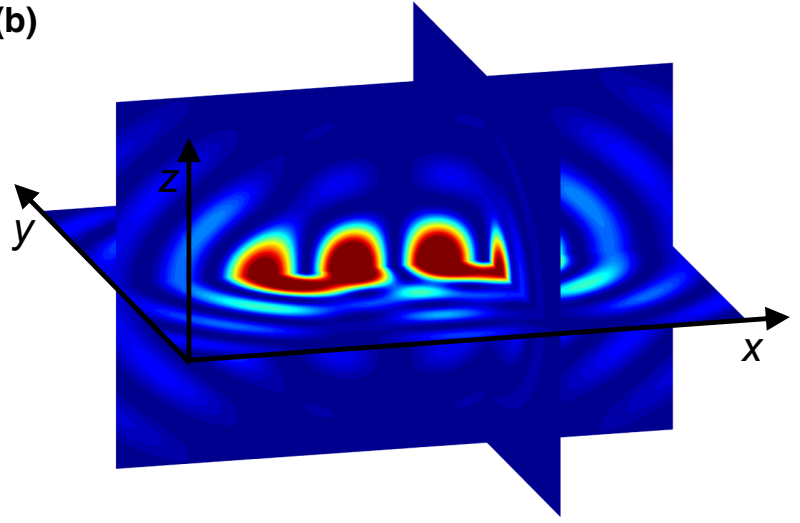

FIG. 4. (a) Local and global coordinates relationship in the spherical coordinate system and (b) the square of the pressure modulus $|p|^{2}$ of two interacting synchronized SBVs of order $(n, m)=(1,1)$ with lateral offset ratio $\delta=2\left(\delta=s / l_{0}\right)$ and no axial offset $\alpha=0\left(\alpha=z_{0} / l_{0}\right)$. Here $l_{0}$ is the distance between the maximum pressure amplitude on the first ring and the origin of a single vortex beam.

where the index $j=1$ denotes the left vortex with beam center $O_{1}(-s, 0,0)$ in global coordinates and $j=$ 2 denotes the lower-right vortex with beam center $\mathrm{O}_{2}\left(s, 0,-z_{0}\right)$, and $\beta_{j}$ is the additional phase angle coming from the temporal term. Here we study synchronized vortices, i.e., vortices with no phase shift: $\beta_{1}=\beta_{2}$. Indeed, the case of nonsynchronized vortices interaction was studied for cylindrical Bessel beams by Gong and Baudoin [28], who showed that optimal conditions are obtained when the two vortices are perfectly synchronized. Since the origin of time can be set arbitrarily, we thus consider $\beta_{1}=\beta_{2}=0$ in the following calculations. The total acoustic field is the superposition of the two SBVs

$$
\begin{aligned}
p= & A_{1} j_{n}\left(k r_{1}\right) P_{n}^{m}\left(\cos \theta_{1}\right) e^{i m \varphi_{1}} \\
& +A_{2} j_{n}\left(k r_{2}\right) P_{n}^{m}\left(\cos \theta_{2}\right) e^{i m \varphi_{2}}
\end{aligned}
$$

where the geometrical relationship of the position and angle coordinates are given in detail in Appendix B 1. The pressure amplitude square $|p|^{2}$ of two synchronized interacting SBVs is illustrated in Fig. 4(b) for a lateral offset ratio $\delta=s / l_{0}=2$ and an axial offset ratio $\alpha=z_{0} / l_{0}=$ 0 , where $l_{0}$ is the distance between the maximum pressure amplitude on the first ring (corresponding to the position of the first maximum of the spherical Bessel function) and the origin of a single vortex beam. In the present simulations, $l_{0}=100 \mu \mathrm{m}$. The maximum pressure amplitudes of the two individual beams are set the same: $\left|p_{\max }\right|=1 \mathrm{MPa}$. See the Supplemental Material [50] (movie 2) for the interference between the two vortices as a function of their offset for different SBV orders. In order to obtain the radiation force exerted by the combination of two SBVs on small particles compared to the wavelength, we must (i) compute the acoustic velocity field associated with the total pressure field given by Eq. (15) with the relation $\mathbf{v}=-i /\left(\rho_{0} \omega\right) \nabla p$, where $\nabla=$ $(\partial / \partial r) \mathbf{e}_{r}+(\partial / r \partial \theta) \mathbf{e}_{\theta}+[\partial /(r \sin \theta) \partial \varphi] \mathbf{e}_{\varphi}$, (ii) compute Gor'kov's potential with Eq. (5) and, finally, (iii) calculate the negative gradient of this potential. The detailed derivation of the velocity field as well as the related derivative relationships are given in Appendix B 2.

\section{B. Particle assembly along the lateral direction}

First, we study the lateral assembly of two particle with two spherical Bessel beams of order $(1,1)$ with no offset in the axial direction (i.e., $z_{0}=0$ ). To enable $3 \mathrm{D}$ assembly, the separate particles should always remain trapped in the axial direction, while being pushed laterally without the possibility for the particles to escape from the potential well. Simulations are performed for two PS particles of radius $a=5 \mu \mathrm{m}$ trapped at the center of two firstorder $[(n, m)=(1,1)]$ SBVs with driving frequency $f=$ $5 \mathrm{MHz}$ and maximum beam amplitude $\left|p_{\max }\right|=1 \mathrm{MPa}$. Slices of the Gor'kov potential in the three planes (i) $(x, y)$, $z=0$, (ii) $(x, z), y=0$, and (iii) $(y, z), x=x_{\text {eq }}$ (defined below) are given in columns 2-4 of Fig. 5, respectively, at different lateral offset ratios in the $x$ direction (along which the particles are assembled), $\delta=3,1$, and 0 [rows (a)-(c)]. The computational domain is $x, y, z \in[-2 \lambda, 2 \lambda]$ with $\lambda=301 \mu \mathrm{m}$ in water. The axial and lateral radiation forces are also given in this figure (first and last columns, respectively) as well as the static equilibrium positions for the two particles denoted with magenta filled circles. Note that the $x$ coordinate of the two equilibrium positions of the particles $\left(x= \pm x_{\mathrm{eq}}\right)$ differ from the position of each individual vortex central axis when $\delta \neq 0$. See the Supplemental Material [50] (movie 3) for the continuous evolution of the Gor'kov's potential and the lateral and axial forces for $\delta$ evolving from 5 to 0 .

As observed from the second row of Fig. 5 and movie 3 , the interference between the two vortices creates a path 

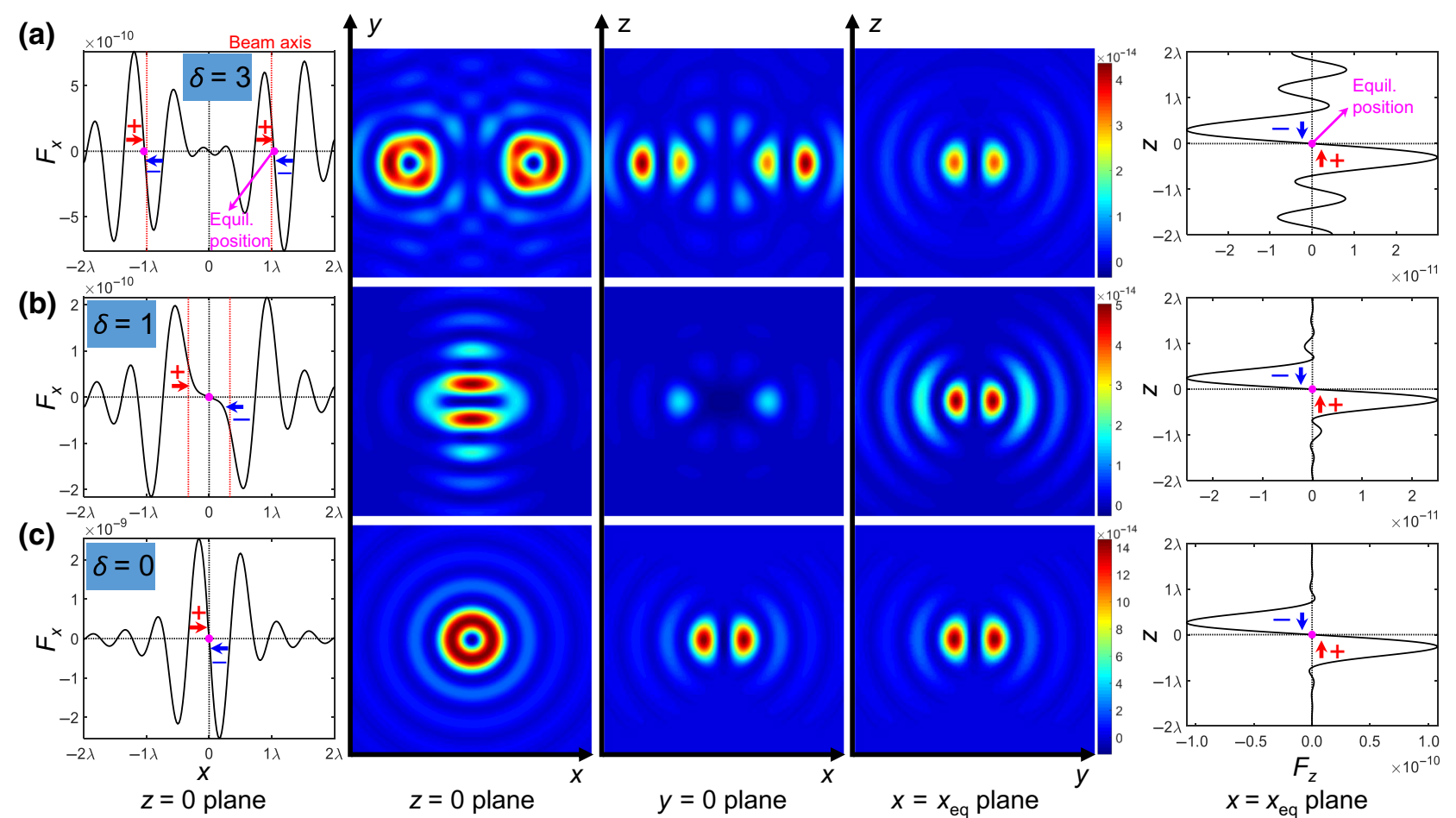

FIG. 5. Particles assembly of particles trapped at the center of two SBVs of order $(1,1)$ along the lateral $(x)$ direction. Columns $2-4$ represent the Gor'kov potential in the $(x, y),(x, z)$, and $(y, z)$ planes, respectively. The lateral and axial radiation forces versus positions are plotted in the first and fifth columns, respectively. The (a)-(c) rows correspond to different offset ratios $\delta$ (dimensionless distance between the vortices core): (a) $\delta=3$; (b) $\delta=1$; (c) $\delta=0$. The particles get trapped at the static equilibrium position indicated by the magenta filled circles in the lateral and axial directions with restoring forces (negative force gradient). The continuous evolution of the Gork'ov potential and the axial and lateral forces as a function of positions with different offset ratios can be seen in movie 3 of the Supplemental Material [50]. The results theoretically demonstrate the ability to laterally assemble particles with synchronized vortices, while maintaining an axial trap.

in the repulsive rings, which enables the assembly of two particles initially trapped individually at the center of the two vortices. All along the way, each of the particles is trapped axially and is pushed by a lateral force along the $x$ axis until the two particles are gathered at the core of the two superimposed vortices [Fig. 5(c)]. This demonstrates the ability for lateral assembly while maintaining an axial trapping with two synchronized SBVs.

\section{Particle assembly along the axial direction}

In three dimensions, both lateral and axial assembly are interesting to assemble complex objects. We now focus on the axial assembly. Hence, we consider two vortices whose central axes coincide, while they are separated by an axial offset ratio $\alpha=z_{0} / l_{0}$. We focus below on the axial trap, but the lateral trap remains effective all along the assembly process. The Gor'kov potential of PS particles trapped in a synchronized SBV field with different axial offset ratios (a) $\alpha=5$, (b) $\alpha=3$, (c) $\alpha=1$, and (d) $\alpha=0$ are given in Fig. 6 . Similarly, the computational region is $x, y, z \in[-2 \lambda, 2 \lambda]$. The Gor'kov potential is symmetric around the $x$ axis (i.e., $x=0)$ in the $(x, z), y=0$ plane, as shown in the colormap panels in Fig. 6(a)-(d). To verify the assembly ability along the axial direction, we calculated the axial radiation force $F_{z}$ as a function of the $z$ position for different offsets. See the Supplemental Material [50] (movie 4) for the continuous evolution of the Gork'ov potential and the axial radiation force as a function of the $z$ position. The results show that two particles are pushed axially until they are assembled, as illuminated from panels (a)-(d). This demonstrates the possibility to assemble particles axially.

\section{CRITICAL SPEED OF MOVING THE TWEEZERS}

The next critical question to address is the speed at which two particles can be assembled (moved). To move particles at a given speed, the acoustic radiation force in the direction of the movement must resist the Stokes' drag acting on the particle. By balancing these two forces, it is possible to determine the critical maximum speed at 
(a)

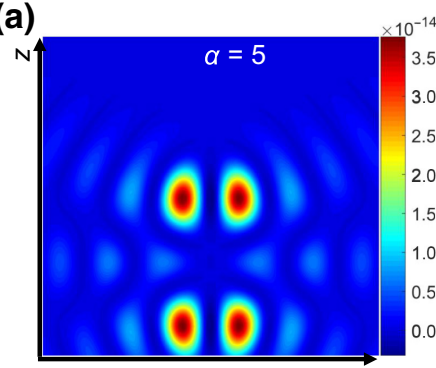

(c)

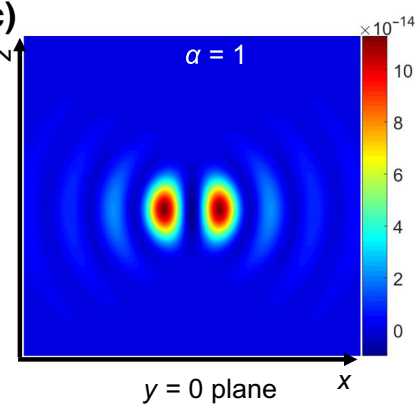

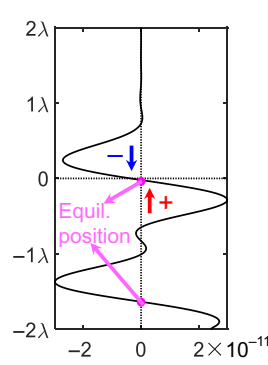

(b)

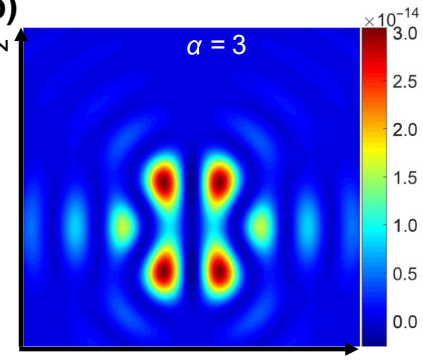

(d)

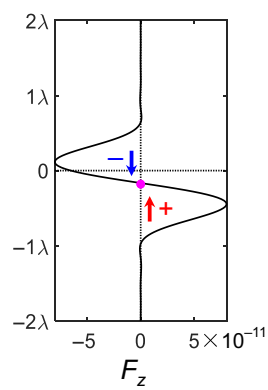

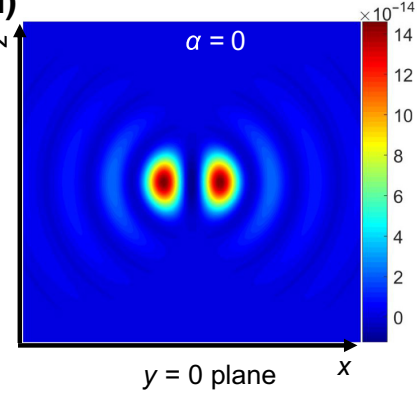
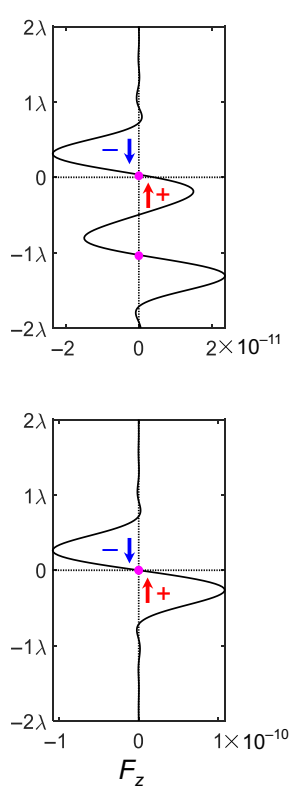

FIG. 6. Particles assembly of particles trapped at the center of two synchronized SBVs of order $(1,1)$ along the axial $(z)$ direction. The Gor'kov potential (color map) and axial force $F_{z}$ are represented for four different axial offset ratios (dimensionless distance between the vortices center) (a) $\alpha=5$, (b) $\alpha=3$, (c) $\alpha=1$, and (d) $\alpha=0$. The continuous evolution of the Gork'ov potential and the axial forces as a function of the $z$ position can be seen in movie 4 of the Supplemental Material [50] for $\alpha$ evolving from 5 to 0.

which acoustical tweezers can be translated without losing the particle. Assuming that the particle is moved at a constant speed, the critical speeds $v_{x}^{\text {cr }}$ and $v_{z}^{\text {cr }}$ in the

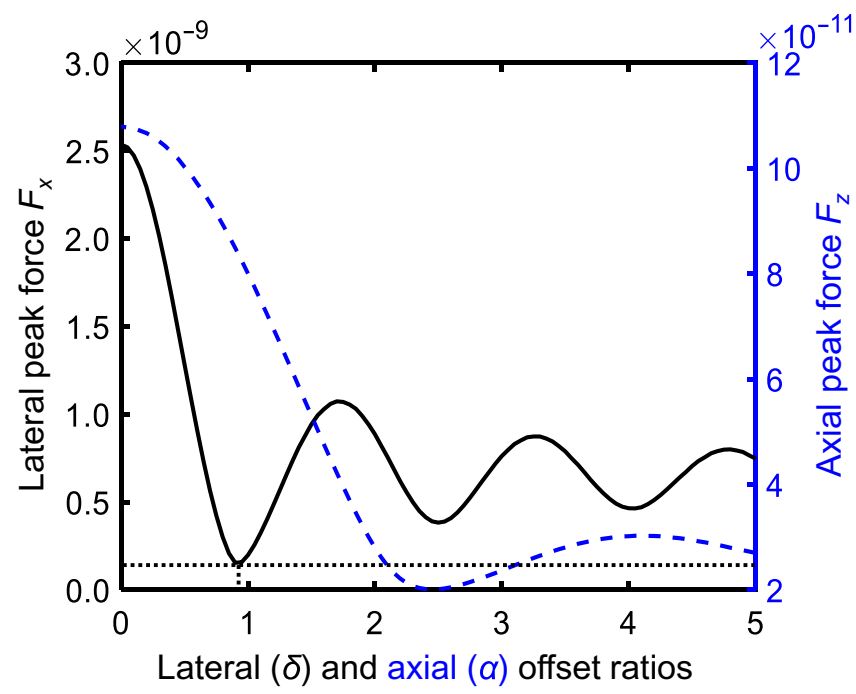

FIG. 7. Lateral $\left(F_{x}\right)$ and axial $\left(F_{z}\right)$ radiation forces (corresponding to the value of the force peak surrounding the static equilibrium position) exerted on a trapped particle in the lateral and axial assembly configurations, respectively, as a function of the dimensionless distance between the particles. For the lateral particles assembly, the critical radiation force $F_{x}^{\mathrm{cr}}=1.538 \times$ $10^{-10} \mathrm{~N}$ is obtained at the lateral offset ratio $\delta=0.9$, while for the axial assembly, the critical radiation force $F_{z}^{\mathrm{cr}}=2.011 \times 10^{-11}$ $\mathrm{N}$ is obtained at the axial offset ratio $\alpha=2.5$. lateral and axial directions, respectively (for lateral and axial assembly), can be determined by balancing the minimum radiation force $\left(F_{x, z}^{\mathrm{cr}}\right)$ along the way (i.e., for different offsets $\delta$ or $\alpha$ ) and the drag force: $\mathbf{F}_{d}=-6 \pi \eta a v_{x, z}^{\mathrm{cr}}$, with $\eta$ the dynamic viscosity. Here the radiation force refers to the radiation force value at the peak surrounding the potential well. Its evolution in the lateral and axial assembly configurations is plotted as a function of the corresponding offset ratios [ $\delta$ for the lateral assembly (black solid line) and $\alpha$ for the axial assembly (blue dashed line)] in Fig. 7. For lateral assembly, the minimum force is obtained for $\delta=0.9$ with the critical radiation force $F_{x}^{\mathrm{cr}}=1.5 \times 10^{-10} \mathrm{~N}$. For axial assembly, the minimum lies at $\alpha=2.5$ with the critical radiation force $F_{z}^{\mathrm{cr}}=2.0 \times 10^{-11} \mathrm{~N}$. The lower force for axial assembly is expected since the axial trap is typically one order of magnitude smaller than the lateral trap for acoustical vortices. We can now compute the critical assembly speed from the relationship $F_{x, z}^{\mathrm{cr}}=F_{d}$, leading to the expression $v_{x, z}^{\mathrm{cr}}=F_{x, z}^{\mathrm{cr}} /(6 \pi \eta a)$. For a PS particle with $a=5 \mu \mathrm{m}$ immersed in water with $\eta=1.0 \mathrm{mPa}$ s, the critical speed for lateral assembly is $v_{x}^{\mathrm{cr}}=1.6 \mathrm{~mm} / \mathrm{s}$, while for axial assembly, we obtain $v_{z}^{\mathrm{cr}}=0.2 \mathrm{~mm} / \mathrm{s}$, values that are compatible with typical operations in microfluidic systems. Note that these values of course depend on the size and composition of the particle and the intensity of the beam. It is interesting to note that the critical speeds scale (i) as the particle radius square $a^{2}$ since the radiation forces $F_{x, z}^{\mathrm{cr}}$ are proportional to $a^{3}$ [see Eq. (3)] and the drag force $F_{d}$ to $a$, and (ii) linearly with the acoustic contrast [also see Eq. (3)]. 


\section{CONCLUSION AND DISCUSSION}

In this work, we have theoretically demonstrated the possibility to assemble in three dimensions (both laterally and axially) particles trapped at the center of two synchronized spherical Bessel acoustical vortices in the long wavelength regime (i.e., for a particle much smaller than the wavelength). The assembly is enabled by the destructive interference between approaching neighboring vortices, which creates an attractive path between the trapped particles. We have also determined the maximum speed at which the particles can be assembled from the balance of the radiation force and the drag force. Speeds of the order of $\mathrm{mm} / \mathrm{s}$ are predicted for $5 \mu \mathrm{m}$ polystyrene spheres, an excitation frequency of $5 \mathrm{MHz}$, and reasonable pressure levels of $1 \mathrm{MPa}$. This study is performed in the analytically tractable case of SBVs trapping small particles compared to the wavelength. Nevertheless, such vortices are difficult to synthesize experimentally since transducers positioned all around the manipulation area would be required. The next step is hence to theoretically and experimentally investigate the assembly of particles with one-sided spherical vortices [13] - i.e., vortices synthesized with a finite aperture lower than $2 \pi$ steradians - beyond the long wavelength approximation. Indeed, it has been shown both theoretically and experimentally that single PS particles can be trapped in three dimensions with these wavefields for particle diameters up to $d \sim 0.3 \lambda$ [14], while simulations predict cells lateral trapping for sizes up to approximately $0.7 \lambda$ [15] (see the supplemental information in Ref. [15]). Such vortices can be synthesized with miniaturized, flat holographic transducers $[15,24]$. We expect that the concept introduced here (assembly with synchronized vortices) might apply for particles beyond the LWR. Nevertheless in such studies, (i) particle resonances might strongly affect particle trapping depending on the constitutive material of both the particles and surrounding fluid, and (ii) the secondary radiation forces [51-53] might also modify the assembly dynamics (while this effect is less important for small-sized particles [52]). Finally, an important question to address in the future is the role of acoustic streaming, including bulk [54 57] and boundary streaming [58,59]. Acoustic streaming could indeed affect the particles and lead to their ejection from the trap, depending on the particle size and composition, the actuation frequency, and the beam topology. Finally, the combination of more than two Bessel beams could be promising to produce desired acoustic traps of special shape with controllable orbital angular momentum [60].

\section{ACKNOWLEDGMENTS}

We acknowledge support from the programs ERC Generator and Prematuration funded by the ISITE Université Lille Nord-Europe (I-SITE ULNE). The authors would like to thank Dr. Udita Ghosh for her careful reading of the manuscript.

\section{APPENDIX A: ACOUSTIC POWER REQUIRED FOR THE SYNTHESIS OF AN ACOUSTIC SPHERICAL BESSEL VORTEX OF ORDER $(n, m)$}

A Bessel spherical vortex can be seen as the interference between an outgoing Hankel spherical vortex of the first kind and a converging Hankel spherical of the second kind:

$$
\begin{aligned}
p & =A_{0} j_{n}(k r) P_{n}^{m}(\cos \theta) e^{i(m \varphi-\omega t)} \\
& =\frac{A_{0}}{2}\left[h_{n}^{(1)}(k r)+h_{n}^{(2)}(k r)\right] P_{n}^{m}(\cos \theta) e^{i(m \varphi-\omega t)} .
\end{aligned}
$$

Of course, since a spherical Bessel vortex is steady over $\mathbf{e}_{r}$, the radial intensity (Poynting) vector I cancels in this direction. Hence, to compute the power required to synthesize a Bessel vortex, only the outgoing or converging wave must be considered. This power can hence be computed by integrating this intensity vector over a sphere of radius $r$ :

$$
\begin{aligned}
\langle\mathcal{P}\rangle & =\iint\langle\mathbf{I}\rangle \cdot \mathbf{n} d S \\
& =\int_{\theta=0}^{\pi} \int_{\varphi=0}^{2 \pi}\left\langle\mathbf{I} \cdot \mathbf{e}_{r}\right\rangle r^{2} \sin \theta d \theta d \varphi
\end{aligned}
$$

with

$$
\begin{gathered}
\langle\mathbf{I}\rangle \cdot \mathbf{e}_{r}=\frac{1}{2} \operatorname{Re}\left[p^{*}\left(\mathbf{v} \cdot \mathbf{e}_{r}\right)\right] \\
\text { and } p=A_{0} h_{n}^{(1)}(k r) P_{n}^{m}(\cos \theta) e^{i(m \varphi-\omega t)} .
\end{gathered}
$$

This integration can be performed in the far field wherein $h_{n}^{(1)}(k r) \approx i^{-(n+1)} e^{i k r} / k r$. First, we obtain

$$
\operatorname{Re}\left[p^{*}\left(\mathbf{v} \cdot \mathbf{e}_{r}\right)\right]=\frac{A_{0}^{2} P_{n}^{m 2}(\cos \theta)}{2 \rho_{0} \omega r^{2}},
$$

whose integration on a sphere in the far field gives

$$
\langle\mathcal{P}\rangle=\frac{\pi A_{0}^{2}}{\rho_{0} k \omega} \frac{2(n+m) !}{(2 n+1)(n-m) !} .
$$

\section{APPENDIX B: COORDINATES RELATIONSHIP AND SYNTHETIC VELOCITIES}

\section{Geometrical relationship of local and global coordinates}

Take Fig. 4(a) as a general case with offsets in the lateral (here $x$ ) and axial $(z)$ directions. The local (position and 
angle) coordinates $\left(r_{1,2}, \theta_{1,2}, \varphi_{1,2}\right)$ are depicted in terms of global spherical coordinates $(r, \theta, \varphi)$ as follows:

$$
\begin{aligned}
& R_{1}^{2}=R^{2}+s^{2}+2 R s \cos \varphi, \\
& R_{2}^{2}=R^{2}+s^{2}-2 R s \cos \varphi, \\
& r_{1}^{2}=R_{1}^{2}+z^{2}=r^{2}+s^{2}+2 r s \sin \theta \cos \varphi, \\
& r_{2}^{2}=r^{2}+s^{2}-2 r s \sin \theta \cos \varphi+2 r \cos \theta z_{0}+z_{0}^{2}, \\
& \cos \theta_{1}=z / r_{1}=r \cos \theta / r_{1}, \\
& \cos \theta_{2}=\left(z+z_{0}\right) / r_{2}=\left(r \cos \theta+z_{0}\right) / r_{2} .
\end{aligned}
$$

Here $R$ and $R_{1,2}$ are radii in cylindrical coordinates. Note that there is no offset in the axial direction when $z_{0}=0$.

\section{Velocity of the synchronized field}

The velocity of the synchronized field could be computed by the vector sum of individual velocities as shown in Eq. (B2) with the pressure expression given in Eq. (14):

$$
\begin{aligned}
\mathbf{v}_{1,2} & =-i \frac{1}{\rho_{0} \omega} \nabla p_{1,2} \\
& =-i \frac{1}{\rho_{0} \omega}\left\{\frac{\partial p_{1,2}}{\partial r} \mathbf{e}_{r}+\frac{1}{r} \frac{\partial p_{1,2}}{\partial \theta} \mathbf{e}_{\theta}+\frac{1}{r \sin \theta} \frac{\partial p_{1,2}}{\partial \varphi} \mathbf{e}_{\varphi}\right\} .
\end{aligned}
$$

The total velocity is

$$
\mathbf{v}=\mathbf{v}_{1}+\mathbf{v}_{2}=v_{r} \mathbf{e}_{\mathrm{r}}+v_{\theta} \mathbf{e}_{\theta}+v_{\varphi} \mathbf{e}_{\varphi} .
$$

The time average of the square of velocity is

$$
\begin{aligned}
\left\langle\mathbf{v}^{2}\right\rangle & =\left\langle\left[v_{r} \mathbf{e}_{r}+v_{\theta} \mathbf{e}_{\theta}+v_{\varphi} \mathbf{e}_{\varphi}\right]^{2}\right\rangle \\
& =\frac{1}{2} \operatorname{Re}\left\{v_{r} v_{r}^{*}+v_{\theta} v_{\theta}^{*}+v_{\varphi} v_{\varphi}^{*}\right\} .
\end{aligned}
$$

To compute the three components of the velocity, the following geometrical and derivative relationships should be applied:

$$
\begin{aligned}
& R_{1,2} e^{i \varphi_{1,2}}=R e^{i \varphi} \pm s, \\
& \partial r_{1} / \partial r=(r+s \sin \theta \cos \varphi) / r_{1}, \\
& \partial r_{1} / \partial \theta=r s \cos \theta \cos \varphi / r_{1}, \\
& \partial r_{1} / \partial \varphi=-r s \sin \theta \sin \varphi / r_{1}, \\
& \partial R_{1} / \partial r=\left(r \sin ^{2} \theta+s \sin \theta \cos \varphi\right) / R_{1}, \\
& \partial R_{1} / \partial \theta=\left(r^{2} \sin \theta \cos \theta+r s \cos \theta \cos \varphi\right) / R_{1}, \\
& \partial R_{1} / \partial \varphi=-r \sin \theta \sin \varphi / R_{1}, \\
& \partial r_{2} / \partial r=\left(r-s \sin \theta \cos \varphi+2 z_{0} \cos \theta\right) / r_{2}, \\
& \partial r_{2} / \partial \theta=-r\left(s \cos \theta \cos \varphi+z_{0} \sin \theta\right) / r_{2}, \\
& \partial r_{2} / \partial \varphi=r s \sin \theta \sin \varphi / r_{2},
\end{aligned}
$$

$$
\begin{aligned}
& \partial R_{2} / \partial r=\left(r \sin ^{2} \theta-s \sin \theta \cos \varphi\right) / R_{2}, \\
& \partial R_{2} / \partial \theta=\left(r^{2} \sin \theta \cos \theta-r s \cos \theta \cos \varphi\right) / R_{2}, \\
& \partial R_{2} / \partial \varphi=r s \sin \theta \sin \varphi / R_{2} .
\end{aligned}
$$

[1] S. Chu, The manipulation of neutral particles, Rev. Mod. Phys. 70, 685 (1998).

[2] S. Chu, J. Bjorkholm, A. Ashkin, and A. Cable, Experimental Observation of Optically Trapped Atoms, Phys. Rev. Lett. 57, 314 (1986).

[3] M. Anderson, J. Ensher, M. Matthews, C. Wieman, and E. Cornell, Observation of bose-einstein condensation in a dilute atomic vapor, Science 269, 198 (1995).

[4] A. Ashkin, J. Dziedzic, and T. Yamane, Optical trapping and manipulation of single cells using infrared laser beams, Nature 330, 769 (1987).

[5] A. Keloth, O. Anderson, D. Risbridger, and L. Paterson, Single cell isolation using optical tweezers, Micromachines 9, 434 (2018).

[6] Y. Liu, D. Cheng, G. Sonek, M. Berns, C. Chapman, and B. Tromberg, Evidence of localized cell heating induced by infrared optical tweezers, Biophys. J. 68, 2137 (1995).

[7] Y. Liu, G. Sonek, M. Berns, and B. Tromberg, Assessing the effects of confinement by 1064-nm laser tweezers using microflurometry, Biophys. J. 71, 2158 (1996).

[8] K. Neuman, E. Chadd, G. Liou, K. Bergman, and S. Block, Characterization of photodamage to Escherichia coli in optical traps, Biophys. J. 77, 2856 (1999).

[9] A. Blasquez, Optical tweezers: Phototoxicity and thermal stress in cells and biomolecules, Micromachines 10, 507 (2019).

[10] F. Crick and A. Hugues, The physical properties of cytoplasm, Exp. Cell Res. 1, 37 (1950).

[11] T. Strick, J.-F. Allemand, D. Bensimon, A. Bensimon, and V. Croquette, The elasticity of a single supercoiled dna molecule, Science 271, 1835 (1996).

[12] S. Schuerle, A. Soleimany, T. Yeh, G. Anand, M. Häberli, H. Fleming, N. Mirkhani, F. Qiu, S. Hauert, X. Wang, B. J. Nelson, and N. Bhatia, Synthetic and living micropropellers for convection-enhanced nanoparticle transport, Sci. Adv. 5, eaav4803 (2019).

[13] M. Baudoin and J.-L. Thomas, Acoustical tweezers for particle and fluid micromanipulation, Annu. Rev. Fluid Mech. 52, $205(2020)$.

[14] D. Baresch, J.-L. Thomas, and R. Marchiano, Observation of a Single-Beam Gradient Force Acoustical Trap for Elastic Particles: Acoustical Tweezers, Phys. Rev. Lett. 116, 024301 (2016).

[15] M. Baudoin, J. Thomas, R. Al Sahely, J. Gerbedoen, Z. Gong, A. Sivery, O. Bou Matar, N. Smagin, P. Favreau, and A. Vlandas, Spatially selective manipulation of cells with single beam acoustical tweezers, Nat. Commun. 11, 4244 (2020).

[16] T. Szabo, in Diagnostic Ultrasound Imaging: Inside Out, edited by A. Press (Academic Press, Oxford, 2014), Chap. 13. 
[17] F. Foster, C. Pavlin, K. Harasiewicz, D. Christopher, and D. Turnbull, Advances in ultrasound biomicroscopy, Ultrason. Med. Biol. 26, 1 (2000).

[18] J. Hulström, O. Manneberg, K. Dopf, H. Hertz, H. Brismar, and M. Wiklund, Proliferation and viability of adherent cells manipulated by standing-wave ultrasound in a microfluidic chip, Ultrason. Med. Biol. 33, 145 (2007).

[19] M. Wiklund, Acoustouidics 12: Biocompatibility and cell viability in microuidic acoustic resonators, Lab Chip 12, 2018 (2012).

[20] M. A. Burguillos, C. Magnusson, M. Nordin, A. Lenshof, P. Augustsson, M. J. Hansson, E. Elmer, H. Lilja, P. Brundin, T. Laurell, et al., Microchannel acoustophoresis does not impact survival or function of microglia, leukocytes or tumor cells, PLoS ONE 8, e64233 (2013).

[21] V. Marx, Biophysics: using sound to move cells, Nat. Meth. 12, 41 (2015).

[22] J. Wu, Acoustical tweezers, J. Acoust. Soc. Am. 89, 2140 (1991).

[23] A. Marzo, S. Seah, B. Drinkwater, D. Sahoo, B. Long, and S. Subramanian, Holographic acoustic elements for manipulation of levitated objects, Nat. Commun. 6, 8661 (2015).

[24] M. Baudoin, J. Gerbedoen, B. M. O. N. Smagin, A. Riaud, and J.-L. Thomas, Folding a focalized acoustical vortex on a at holographic transducer: miniaturized selective acoustical tweezers, Sci. Adv. 5, eaav1967 (2019).

[25] D. Baresh, J.-L. Thomas, and R. Marchiano, Spherical vortex beams of high radial degree for enhanced single-beam tweezers, J. Appl. Phys. 113, 184901 (2013).

[26] C. Courtney, C. Demore, H. Wu, A. Grinenko, P. Wilcox, S. Cochran, and B. Drinkwater, Independent trapping and manipulation of microparticles using dexterous acoustic tweezers, Appl. Phys. Lett. 104, 154103 (2014).

[27] A. Marzo and B. Drinkwater, Holographic acoustic tweezers, Proc. Natl. Acad. Sci. 116, 84 (2019).

[28] Z. Gong and M. Baudoin, Particle Assembly with Synchronized Acoustical Tweezers, Phys. Rev. Appl. 12, 024045 (2019).

[29] B. Hefner and P. Marston, An acoustical helicoidal wave transducer with applications for the alignment of ultrasonic and underwater systems, J. Acoust. Soc. Am. 106, 3313 (1999).

[30] J.-L. Thomas and R. Marchiano, Pseudo Angular Momentum and Topological Charge Conservation for Nonlinear Acoustical Vortices, Phys. Rev. Lett. 91, 244302 (2003).

[31] K. Volke-Sepulveda, A. Santillan, and R. Boullosa, Transfer of Angular Momentum to Mfrom Acoustical Vortices in Free Space, Phys. Rev. Lett. 100, 024302 (2008).

[32] K. Skeldon, C. Wilson, M. Edgar, and J. Padgett, An acoustic spanner and its associated rotational doppler shift, New J. Phys. 10, 013018 (2008).

[33] A. Riaud, J. Thomas, E. Charron, A. Bussoniére, and O. Bou Matar, Anisotropic Swirling Surface Acoustic Waves from Inverse Filtering for On-Chip Generation of Acoustic Vortices, Phys. Rev. Appl. 4, 034004 (2015).

[34] A. Riaud, M. Baudoin, O. Thomas, and J.-L. Bou Matar, Saw synthesis with idts array and the inverse filter: toward a versatile saw toolbox for microfluidics and biological applications, IEEE Trans. Ultrason. Ferroelectr. 63, 1601 (2016).

[35] S. Gspan, A. Meyer, S. Bernet, and M. Ritsch-Marte, Optoacoustic generation of a helicoidal ultrasonic beam, J. Acoust. Soc. Am. 115, 1142 (2004).

[36] J. Elao, J. Prietao, and F. Seco, Airborne ultrasonic vortex generation using flexible ferroelectrets, IEEE Trans. Ultrason. Ferroelectr. Freq. Control 58, 1651 (2011).

[37] N. Jimenez, R. Pico, V. Sanchez-Morcillo, V. RomeroGarcia, L. Garcia-Raffi, and K. Staliunas, Formation of high order acoustic bessel beams by spiral diffraction gratings, Appl. Rev. E 94, 053004 (2016).

[38] X. Jiang, Y. Li, B. Liang, J.-C. Cheng, and L. Zhang, Convert acoustic resonances to orbital angular momentum, Appl. Rev. Lett. 117, 034301 (2016).

[39] X. Jiang, J. Zhao, S. Liu, X. Zou, J. Yang, C.-W. Qiu, and J. Cheng, Broadband and stable acoustic vortex emitter with multi-arm coiling slits, Appl. Phys. Lett. 108, 203501 (2016).

[40] C. Naify, C. Rohde, T. Martin, M. Nicholas, M. Guild, and G. Orris, Generation of topologically diverse acoustic vortex beams using a compact metamaterial aperture, Appl. Phys. Lett. 108, 223503 (2016).

[41] T. Wang, M. Ke, W. Li, Q. Yang, C. Qiu, and Z. Liu, Particle manipulation with acoustic vortex beam induced by a brass plate with spiral shape structure, Appl. Phys. Lett. 109, 123506 (2016).

[42] A. Riaud, M. Baudoin, O. Bou Matar, L. Becerra, and J.-L. Thomas, Selective Manipulation of Microscopic Particles with Precursors Swirling Rayleigh Waves, Phys. Rev. Appl. 7, 024007 (2017).

[43] M. Terzi, S. Tsysar, P. Yuldashev, M. Karzova, and O. Sapozhnikov, Generation of a vortex ultrasonic beam with a phase plate with an angular dependence of the thickness, Moscow Univ. Phys. Bull. 72, 61 (2017).

[44] N. Jimenez, V. Romero-Garcia, L. Garcia-Raffi, F. Camarena, and K. Staliunas, Sharp acoustic vortex focusing by fresnel-spiral zone plates, Appl. Phys. Lett. 112, 204101 (2018).

[45] R. Muelas-Hurtado, J. Ealo, J. Pazos-Ospina, and K. VolkeSepulveda, Generation of multiple vortex beam by means of active diffraction gratings, Appl. Phys. Lett. 112, 084101 (2018)

[46] S. Jimenez-Gambin, N. Jimenez, J. Benlloch, and F. Camarena, Generating bessel beams with broad depth-offield by using phase-only acoustic holograms, Sci. Rep. 9, 20104 (2019).

[47] X.-D. Fan and L. Zhang, Trapping Force of Acoustical Bessel Beams on a Sphere and Stable Tractor Beams, Phys. Rev. Appl. 11, 014055 (2019).

[48] L. Gor'kov, On the forces acting on a small particle in an acoustical field 49. in an ideal fluid, Sov. Phys. Dokl. 6, 773 (1962).

[49] N.B. The factor of 4 instead of 2 in the potential and kinetic energies comes from the fact that we consider the square of the modulus of the complex expressions of the pressure and velocity, which are equal to 2 times the time average of the square of the real expressions of the pressure and velocity fields. 
[50] See Supplemental Material at http://link.aps.org/supplemen tal/10.1103/PhysRevApplied.14.064002 for further details on acoustic fields and radiation forces.

[51] A. Doinikov, Acoustic radiation interparticle forces in a compressible fluid, J. Fluid Mech. 444, 1 (2001).

[52] G. Silva and H. Bruus, Acoustic interaction forces between small particles in an ideal fluid, Phys. Rev. E 90, 063007 (2014).

[53] M. Wang, C. Qiu, S. Zhang, R. Han, M. Ke, and Z. Liu, Sound-mediated stable configurations for polystyrene particles, Phys. Rev. E 96, 052604 (2017).

[54] C. Eckart, Vortices and streams caused by sound waves, Phys. Rev. 73, 68 (1948).

[55] A. Anäuser, R. Wunenburger, and E. Brasselet, Acoustic Rotational Manipulation Using Orbital Angular Momentum Transfer, Phys. Rev. Lett. 109, 034301 (2012).
[56] A. Riaud, M. Baudoin, J. Thomas, and O. Bou Matar, Cyclones and attractive streaming generated by acoustical vortices, Phys. Rev. E 90, 013008 (2014).

[57] D. Baresch, J. Thomas, and R. Marchiano, Orbital Angular Momentum Transfer to Stably Trapped Elastic Particles in Acoustical Vortex Beams, Phys. Rev. Lett. 121, 074301 (2018).

[58] L. Rayleigh, On the circulation of air observed in kundt's tubes, and on some allied acoustical problems, Phil. Trans. R. Soc. Lond. 175, 1 (1884).

[59] J. Karlsen, W. Qiu, P. Augustsson, and H. Bruus, Acoustic Streaming and its Suppression in Inhomogeneous Fluids, Phys. Rev. Lett. 120, 054501 (2018).

[60] A. Kovalev and V. Kotlyar, Orbital angular momentum of superposition of identical shifted vortex beams, J. Opt. Soc. Am. A Opt. Image Sci. Vis. 32, 1805 (2015). 